\title{
Rising to the climate challenge
}

\author{
The award of a Nobel prize to an advisory body in the science of climate change rightly reflects the \\ organization's many virtues, and should spur it on in its mission to assess and address global warming.
}

T he Intergovernmental Panel on Climate Change (IPCC) is not a household name but it deserves to be. Its important and altruistic work during the past two decades fully merits this year's Nobel Peace Prize, which it shares with the former US vice-president Al Gore (see page 766).

The current understanding of anticipated climate change and its effect on ecosystems and societies, uncertainties and all, is not anecdotal. Rather, it is articulated explicitly as a consensus view of a worldwide community of researchers. Too few politicians and members of the public appreciate this. And although not every individual scientist involved will fully agree with each sentence and each probability estimate in the IPCC's reports, few if any will seriously question that what the IPCC delivers is as good a chunk of scientific advice on climate change as anyone could hope to get.

In its latest, fourth assessment, a synthesis of which will be released next month, the IPCC has compiled the strongest evidence so far that the current warming trend is the increasingly dangerous result of human activity. This is an apolitical statement. Taking the appropriate political steps is the responsibility of the countries that, in Bali in December, will continue negotiating a follow-up agreement to the 1997 Kyoto Protocol on climate change.

The challenge of climate change urgently demands a coherent political response. But no matter whether and how soon an agreement on stabilizing greenhouse-gas emissions at safe levels might be reached, the IPCC should continue its successful work in informing policy-makers on how the science is developing, and on further climate signals and trends as they are uncovered.

Even if policy-makers can mitigate the effects of climate change, adaptation to them will still be a necessity. But how much, and when, and where? Perhaps the biggest challenge for the climate-research community over the next five years will be to use new scenarios of mitigation and adaptation to generate predictions about how climate change will affect specific regions. Climate modellers are already gearing up to address the problem of predicting regional climate change out to 2030 or so. The results of their increasingly detailed projections are likely to become the core of a fifth IPCC assessment in five or six years.

Although much of the IPCC's strength lies in the very scale and thoroughness of its assessments, reports of more restricted scope may also be desirable in the shorter term. There are a number of specific threats that merit deeper assessment, such as the risks of rising sea level and retreating sea-ice in the Arctic, and the effects of feedback loops on the climate system.

There are other important issues that would also benefit from being reviewed promptly by the IPCC. The economic costs of mitigation scenarios, including
"The challenge of climate change urgently demands a coherent political response." doing nothing, should be addressed, as should the vexed but persistent debates surrounding engineered attempts to influence the climate system.

Many climate scientists would like to move away from an IPCC process in which three independent working groups that investigate science, impacts and mitigation, respectively, work almost entirely independently of each other. But the established process is difficult to avoid in drawing up a full-scale assessment, and any suggestion of a merger should be resisted: assessing mitigation is best kept separate from assessing science if only to support the objectivity of the latter. More focused studies can involve greater interaction between, say, climate modellers, impact researchers, economists and coastal engineers.

Climate science - both measurement and modelling - will develop rapidly over the next few years, and alarm may grow with further insights. But a fifth assessment by the IPCC should not be rushed. This Nobel peace laureate is an organization whose strengths include an understanding that, however urgent the challenge, robust scientific advice, like science itself, needs patience.

\section{Do-it-yourself science}

\section{How much involvement can patient advocates have in genetics?}

S ome of the hype that accompanied the first publications of the human genome sequence in 2001 may have worn off. But these are still heady times for genomics, as demonstrated this week by the release of a greatly enhanced haplotype map or HapMap, which describes the most common forms of human genetic variation (see page 851).

The map builds on an earlier version published in 2005. It may, for example, shed some light on aspects of the genome that help to account for certain differences between people of different geographical origins (see page 762). There have been plenty of other research findings this year that demonstrate the power of genomics to deliver clues that could yield better medicine, including studies based on the HapMap that have uncovered lists of multiple genes that may be associated with the risk of developing specific diseases.

But there remain relatively few examples where this has led to better treatment options for patients and doctors. The difficulties of selecting relevant gene and protein markers, and then developing them into marketable tests that doctors will use, remain formidable (see page 770). And for patients, doctors and even some geneticists, there is growing frustration at the lack of clarity in some research 